\title{
Análisis del ajuste de mascarillas autofiltrantes en combinación con las mascarillas quirúrgicas para la protección del profesional sanitario en su atención a pacientes afectos de SARS-COV-2 a partir de un estudio experimental
}

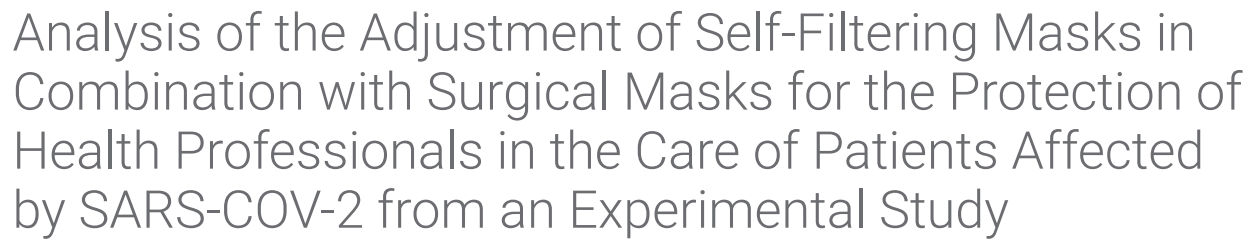

Analysis of the Adjustment of Self-Filtering Masks in Combination with Surgical Masks for the Protection of Health Professionals in the Care of Patients Affected by SARS-COV-2 from an Experimental Study

Raquel Garcia Vela ${ }^{1}$

Rosa Cabanas Valdés² (] 0000-0002-5255-2494

Santos Huertas Ríos ${ }^{1}$

Rafel Donat Roca ${ }^{3}$ (1) 0000-0001-6699-6857

Pol Monné Cuevas ${ }^{4}$ (i] 0000-0001-9884-8189

Luciana Moizé Arcone ${ }^{5}$ @ 0000-0001-6656-9089

Jordi Calvo Sanz ${ }^{4,5}$ @ 0000-0002-6860-6725

${ }^{1}$ Centro de Innovación e Investigación de la Dirección de Prevención (DPV) - Laboratorio de Higiene Industrial. Hospital -Centro de Prevención y Rehabilitación Asepeyo St. Cugat del Vallés. Barcelona. España.

2Departamento de Fisioterapia, Facultad de Medicina y Ciencias de la Salud, Universitat Internacional de Catalunya (UIC-Barcelona), Barcelona, España.

${ }^{3}$ Facultad de Ciencias de la Salud UManresa. Grupo de Investigación en Podología, Biomecánica, Fisioterapia y Ejercicio Terapéutico (GRIBIPOFET), Universidad de Vic-Universitat Central de Catalunya. Manresa, España.

${ }^{4}$ Departamento de Rehabilitación, Hospital -Centro de Prevención y Rehabilitación Asepeyo St.Cugat del Vallés. Barcelona. España.

${ }^{5}$ Departamento de Fisioterapia,Escuela Superior de Ciencias de la Salud- TecnoCampus Mataró-TCM (Universitat Pompeu Fabra - UPF), Mataró. España.

Fechas · Dates

Recibido: 2020.11.20

Aceptado: 2021.02.10

Publicado: 2021.04.15
Correspondencia · Corresponding Author

Dr. Jordi Calvo Sanz

jcalvosanz@gmail.com 


\title{
Resumen
}

Introducción: Un componente importante del equipo de protección individual (EPI) frente al SARS-CoV-2 son las mascarillas quirúrgicas y las mascarillas autofiltrantes (FFP). La norma europea EN 149 establece y clasifica las mascarillas autofiltrantes en tres niveles de protección dependiendo del porcentaje de fuga del total de partículas en suspensión del aire exterior hacia el aire interior FFP1, FFP2, FFP3. El objetivo de este e ensayo de laboratorio es determinar y cuantificar el nivel de ajuste de las mascarillas autofiltrantes FFP2 combinadas con las mascarillas quirúrgicas mediante series de pruebas de ajuste (fit test).

Material y Métodos: Se utilizó el equipo medidor de ajuste de mascarillas FFP modelo PortaCount ${ }^{\circledR}$ Pro +8038 compatible con las normas y metodología de la OSHA (Occupational Safety and Health Administration) de los EEUU. Se realizaron series de pruebas de ajuste sobre diferentes modelos de mascarillas autofiltrantes FFP2 con y sin mascarilla quirúrgica para diferentes situaciones de respiración del trabajador participante en este experimento.

Resultados: El uso de la mascarilla quirúrgica sobre una mascarilla autofiltrante FFP2 aporta una mejora en la protección respiratoria determinante, incrementando el factor de ajuste hasta de +200 (el factor de ajuste mínimo debe ser 100).

Conclusiones: Las mascarillas quirúrgicas cuando se usan conjuntamente con las mascarillas autofiltrantes, podrían mejorar significativamente el grado de ajuste de todas las mascarillas autofiltrantes proporcionando una mayor eficacia de filtración y una mayor protección al usuario frente a la exposición a aerosoles.

Palabras clave: COVID-19; mascarilla quirúrgica; enfermedades infecciosas; SARS-CoV-2; mascarilla autofiltrante; FFP2, factor de ajuste.

\begin{abstract}
Introduction: Frontline healthcare workers have a high risk of exposure to SARS-CoV-2 coronavirus, which causes COVID-19. The use of appropriate personal protective equipment (PPE) is essential to prevent this occupational disease. Surgical masks and filtering face piece (FFP) respirators are important parts of this PPE. European standard EN 149 establishes three protection levels for FFP respirators (FFP1, FFP2, FFP3), depending on the particle infiltration degree through their materials, and these, in turn, are based on their filtration effectiveness. The aim of this laboratory test is to determine and quantify the filtration and fit rate of different FFP respirators, singly and in combination with surgical masks, by performing a series of fit tests and consequently, to check whether this combination improves protection levels for healthcare workers who care for COVID-19 patients.
\end{abstract}

Material and Methods: Several FFP respirators and surgical masks, singly and in combination, were fit tested with a PortaCount Pro +8038 , which fulfills OSHA standards, in a series of tests performed on healthcare workers in seven different breathing situations when taking care on COVID-19 patients, in order to determine and quantify their fit to the workers' face.

Results: Wearing a surgical mask together with a highly efficient FFP respirator provided increased respiratory protection. Interestingly, one of these highly efficient FFP models, combined with a surgical mask, achieved a protection factor over 200 (whereas 100 is the minimum required protection factor). 
Conclusions: Surgical masks, when used together with a FFP2 respirator, could significantly improve the degree of fit of all self-filtering face piece by providing greater filtration efficiency and greater user protection from exposure to aerosols.

Keywords: COVID-19; surgical masks; infectious disease; SARS-CoV-2; Respirator; filtering face piece (FFP); fit test; protection factor.

\section{Introducción}

El COVID-19 es una enfermedad infecciosa causada por el coronavirus SARSCoV-2(1). Según las pruebas actuales, este virus se transmite principalmente entre las personas a través de aerosoles. Las personas son más infecciosas en el período inicial después de la infección, donde es común tener pocos o ningún síntoma (2-6) . El abrumador número de personas infectadas no sólo provoca en muchas ocasiones el cierre generalizado de la comunidad, sino que también colapsa los sistemas de atención de la salud y pone a los trabajadores de la salud en un posible riesgo. El virus del SARS-CoV-2 se transmite principalmente, entre las personas, por vía aérea a través de aerosoles. La comunidad científica apunta la posibilidad de que ésta sea la forma de transmisión dominante, y más habitual de contagio en eventos de super-propagación según el informe científico sobre vías de transmisión SARS-CoV-2 del Ministerio de Ciencia e Innovación de España con fecha 29.10.2020. La transmisión por aerosoles se da también en circunstancias y entornos específicos en los que se realizan procedimientos o tratamientos de apoyo que generan aerosoles con presencia de gotículas de $<5 \mu \mathrm{m}$ de diámetro, como pueden ser, traqueotomía, intubación endotraqueal y ventilación manual previa, broncoscopia, aspiración abierta, administración de tratamiento nebulizado, giro del paciente a la posición prona, desconexión del paciente del ventilador, ventilación no invasiva con presión positiva y reanimación cardiopulmonar. En estos casos será necesaria la protección con mascarilla autofiltrante FFP2 o FFP3, en su defecto podría ser mascarilla FFP2 + mascarilla quirúrgica.

En el estado actual de la pandèmia de la COVID-19 es esencial practicar medidas para prevenir la transmisión del virus. Éstas consisten en la higiene de las manos, el uso de EPI, el distanciamiento físico y el respeto de los protocolos para la reducción de emisión de gotes durante la respiración y la tos ${ }^{(7)}$. Los trabajadores sanitarios requieren de los equipos de protección individual (EPI) para evitar que se contaminen ellos mismos y a su vez que propagen la enfermedad( ${ }^{(8,9)}$. Los EPI son particularmente importantes para disminuir el riesgo ocupacional de infección respiratoria cuando no se dispone de vacunaciones o tratamientos antiinfecciosos específicos ${ }^{(10)}$. Los EPI consisten en: a) mascarillas autofiltrantes FPP o piezas faciales filtrantes, mascarillas quirúrgicas o médicas, b) protección ocular: las pantallas faciales o las gafas protectoras, c) protección de las manos: los guantes, d) protección del cuerpo: las batas de manga larga resistentes al agua y los cubre-zapatos ${ }^{(11-13)}$. 
Para los procedimientos que generan aerosoles, las mascarillas N95 se recomiendan unánimemente en las directrices nacionales e internacionales; sin embargo, hay inconsistencias en las recomendaciones para la atención rutinaria y los procedimientos que no generan aerosoles de los pacientes de COVID-19. La evidencia de una revisión sistemàtica ${ }^{(10)}$ está en consonancia con las recomendaciones actuales de la Organización Mundial de la Salud (OMS) y la Agencia de Salud Pública del Canadá de utilizar mascarillas médicas para los procedimientos que no generan aerosoles cuando se atiende a los pacientes de COVID-19. Por el contrario, los Centros de Control y Prevención de Enfermedades (CDC) y el Centro Europeo para la Prevención y el Control de las Enfermedades (ECDC) recomienda el uso de mascarillas N95 para procedimientos que no generen aerosoles en lugar de las mascarillas médicas, que son más baratas y están más disponibles.

Las mascarillas quirúrgicas están diseñadas para evitar propagar partículas infecciosas, es decir, para evitar que el que las lleva contagie a otras personas. No son auténticos EPIs, ya que no reúnen la condición inherente a un EPI de proteger a quien lleva puesto dicho dispositivo. Las mascarillas quirúrgicas evitan la emisión al ambiente exterior de los propios aerosoles, gotículas y gotas generados por quien lleva puesta la mascarilla, contribuyendo como medida de protección sanitaria a la no propagación del agente biológico en la población. Por eso los controles de calidad se hacen de dentro hacia fuera. Las mascarillas quirúrgicas no se ajustan a la cara, como sí lo hacen las mascarillas autofiltrantes cuando su uso es correcto. Eso implica que un porcentaje del aire inhalado o exhalado no pasará por el filtro, sino que lo hará a través de la superficie de contacto de la mascarilla con la cara (sin filtrar).

Los estudios de laboratorio han mostrado que, efectivamente, las mascarillas quirúrgicas retienen un porcentaje menor de las partículas pequeñas (menores de una micra) que las N95 y FFP2. Además, el porcentaje retenido probablemente sería aún menor al toser o al estornudar, porque la velocidad del aire es mayor que la velocidad a la que se suelen hacer los experimentos (en general, cuanto mayor es la velocidad, más partículas logran atravesar el filtro).

Existen diferentes categorías de mascarillas quirúrgicas y autofiltrantes para los trabajadores sanitarios europeos según el European Centre for Disease Prevention and Control $(E C D C)^{(13)}$. Tienen estándares rigurosos que evalúan las mascarillas quirúrgicas utilizadas en los entornos sanitarios, es decir, la capacidad de la mascarilla para proteger al usuario de las partículas infecciosas. Una mascarilla facial quirúrgica es un dispositivo médico que cubre la boca, la nariz y la barbilla asegurando una barrera que limita la transición de un agente infeccioso entre el personal del hospital y el paciente ${ }^{(14)}$.

Se ha demostrado que la aplicación de mascarillas quirúrgicas como control de la fuente disminuye la liberación de gotas y salpicaduras respiratorias portadoras de virus respiratorios ${ }^{(15)}$ y se recomiendan para reducir la transmisión de la tuberculo$\operatorname{sis}^{(16)}$ y la gripe ${ }^{(17-19)}$. Sin embargo, según las circunstancias, no protege de la inhalación de las partículas muy pequeñas suspendidas en el aire que potencialmente son portadoras del virus. Hay tres tipos diferentes de mascarillas quirúrgicas: tipo 
I: eficacia de filtrado de bacterias $>95 \%$, tipo II: eficacia de filtrado de bacterias > $98 \%$ y tipo IIR: eficacia de filtrado de bacterias $>98 \%$ y resistente a las salpicaduras. La mascarilla quirúrgica está diseñada para un solo uso, debe ser cambiada tan pronto como se humedezca y al menos cada 4 horas, teniendo en cuenta las condiciones de uso y la integridad.

Las mascarillas autofiltrantes están diseñadas para proteger al usuario de la exposición a los contaminantes (partículas sólidas o líquidas en suspensión) del aire a través de la filtración y el ajuste, correctamente ajustadas proporcionan una mejor protección en comparación con las mascarillas quirúrgicas. Cumplen en Europa con los requisitos definidos en el Reglamento (UE) 2016/425 a través de la Norma Europea EN 149:2001+A1:2009(20). En otros países con normas similares como NIOSH-42CFR84 en los Estados Unidos o GB2626-2006 en China. La norma EN 149:2001-A1:2010(21) establece tres niveles de protección en función de la fuga de todas las partículas al interior, ya sea por el ajuste de la mascarilla a la cara o por la penetración a través del filtro, siempre medido según la medición aritmética de las pruebas de laboratorio realizadas por los portadores. Estas son: a) mascarillas FFP1 que filtran al menos el $80 \%$ de los aerosoles (fuga total hacia el interior $<22 \%)$, b) mascarillas FFP2 que se llevan en el rostro de los portadores, formando un sello hermético alrededor de la nariz y la boca (el equivalente en Estados Unidos es N95) mascarillas que filtran al menos el $94 \%$ de los aerosoles (fuga total hacia el interior $<8 \%$ ) y c) mascarillas FFP3 que filtran al menos el $99 \%$ de los aerosoles (fuga total hacia el interior $<2 \%)^{(22)}$. Algunas mascarillas tienen válvulas de exhalación que tienden a mantener la cara más fresca y previenen la acumulación de humedad. La mascarilla plegada autofiltrante con carbón activado para partículas, pinturas y olores fuertes FFP2 con válvula lateral facilita la respiración y reduce el calor y la humedad dentro de la mascarilla, además de mejorar la comunicación. Las mascarillas con válvula de exhalación no deben utilizarse cuando se trabaja en condiciones estériles ${ }^{(23)}$.

El ECDC y la OMS afirman que si el personal sanitario va a realizar procedimientos generadores de aerosoles como la recogida de muestras, o la intubación en un entorno COVID-19, deben utilizar mascarillas FFP2/FFP3 que proporcionen una protección de alto nivel(8,10,11,20). El tiempo de uso debe estar de acuerdo con las instrucciones de uso ${ }^{(24)}$.

El objetivo de este e ensayo de laboratorio es determinar y cuantificar el nivel de ajuste de las mascarillas autofiltrantes FFP2 combinadas con las mascarillas quirúrgicas mediante series de pruebas de ajuste (fit test).

\section{Material y Métodos}

Se llevó a cabo un estudio experimental, realizando un ensayo y prueba de laboratorio valorando el nivel de ajuste de la mascarilla quirúrgica en combinación con una mascarilla autofiltrante. Se realizaron una serie de pruebas, calculando el valor obtenido factor de ajuste or fit test aportado directamente por el equipo. La ubicación del experimento se llevó a cabo en la sala de higiene industrial del Centro 
de Innovación e Investigación de la Dirección de Prevención (DPV) ubicado en el Hospital -Centro de Prevención y Rehabilitación ASEPEYO Sant Cugat del Vallés (Barcelona, España).

Se establecieron los siguientes criterios de inclusión y exclusión de trabajadores voluntarios para portar los distintos modelos de mascarillas autofiltrantes y quirúrgicas objeto de las pruebas de ajuste.

-Criterios de inclusión:

- Que tengan un buen estado de salud

- Mayor de edad

- Que no hayan padecido ni tengan ninguna patología respiratoria asociada -Criterios de exclusión:

- Haber contraído la Covid-19 (se requirió prueba PCR frente a SARS-CoV-2 y test serológico negativo).

Se ha utilizado un método de análisis cuantitativo del factor de ajuste que permite evaluar la adecuación de la mascarilla autofiltrante. Esta debe ser adecuada a la fisonomía, al rostro, y debe ajustarse perfectamente para evitar que el aire que pueda estar contaminado pase entre nuestra cara y la mascarilla, eludiendo de esta manera el material de filtración, el cual evitaría la exposición al riesgo.

La prueba de ajuste está basada en un protocolo de la OSHA (Occupational Safety and Health Administration. United States. Department of Labor) para evaluar cualitativa o cuantitativamente la eficacia de ajuste de mascarillas autofiltrantes.

El equipo utilizado ha sido el instrumento medidor PortaCount ${ }^{\circledR}$ Pro+ Respirator Fit Tester 8038. Dispone de las pertinentes certificaciones de calibrado (Anexo 1), y es el elegido como instrumento modelo para la medición de ajuste según el procedimiento OSHA 29 CFR 1910.134. El PortaCount Pro+ Fit Tester es un método de prueba de ajuste que cumple con las normas de HSE (Reino Unido) y OSHA (EE.UU.).

El instrumento mide la concentración de partículas en el aire. Por un lado la del ambiente exterior a la mascarilla autofiltrante y por otro lado, la del ambiente interior a ésta. Posteriormente se comparan ambas concentraciones y al resultado de realizar su cociente se denomina factor de ajuste. Este es una estimación cuantitativa del ajuste de una mascarilla autofiltrante particular para cada individuo. El instrumento realiza directamente el cálculo del factor de ajuste.

El factor de ajuste de la mascarilla se calcula mediante la relación entre el número de partículas presentes en el recinto donde se realizan las pruebas de ajuste y el número de partículas en el interior de la mascarilla del trabajador que es sometido a tales pruebas. El factor de ajuste mínimo de una mascarilla autofiltrante debe ser 100, que significa que el aire del interior de la mascarilla tiene 100 veces menos partículas que el aire del exterior de ésta. 


\section{Factor de Ajuste $=\frac{\text { Concentración exterior }}{\text { Concentración interior }}$}

Para medir las partículas el equipo tiene un doble tubo de muestreo: uno obtiene muestras del aire ambiente (azul) y el otro se conecta a la mascarilla de respiración (blanco) y realiza una muestra desde el interior de la misma. El aerosol se introduce en el equipo con la ayuda de una bomba de aspiración haciéndolo pasar por el puerto de muestreo ambiente (azul) o el de muestreo de la mascarilla (blanco). La válvula de conmutación determina el puerto que se utiliza. El sensor de PortaCount Pro® se compone de un saturador, un condensador y elementos ópticos en la cámara de detección. El saturador está revestido de una mecha empapada de alcohol isopropílico que moja las partículas del aerosol, saturado de vapor que, a continuación, pasa al tubo del condensador.

El vapor de alcohol se condensa en las partículas y hace que se conviertan en gotas, permitiendo que el detector pueda contabilizarlas al aumentar su tamaño. A continuación, las gotas pasan por una boquilla y se introducen en la cámara de detección. La óptica del sensor se compone de un diodo láser y una serie de lentes que enfocan la luz del láser en la cámara de detección justo por encima de la boquilla. Cada partícula que pasa dispersa la luz, la óptica capta dicha luz y la lleva a un fotodetector, el cual genera un impulso eléctrico (Figura 1).
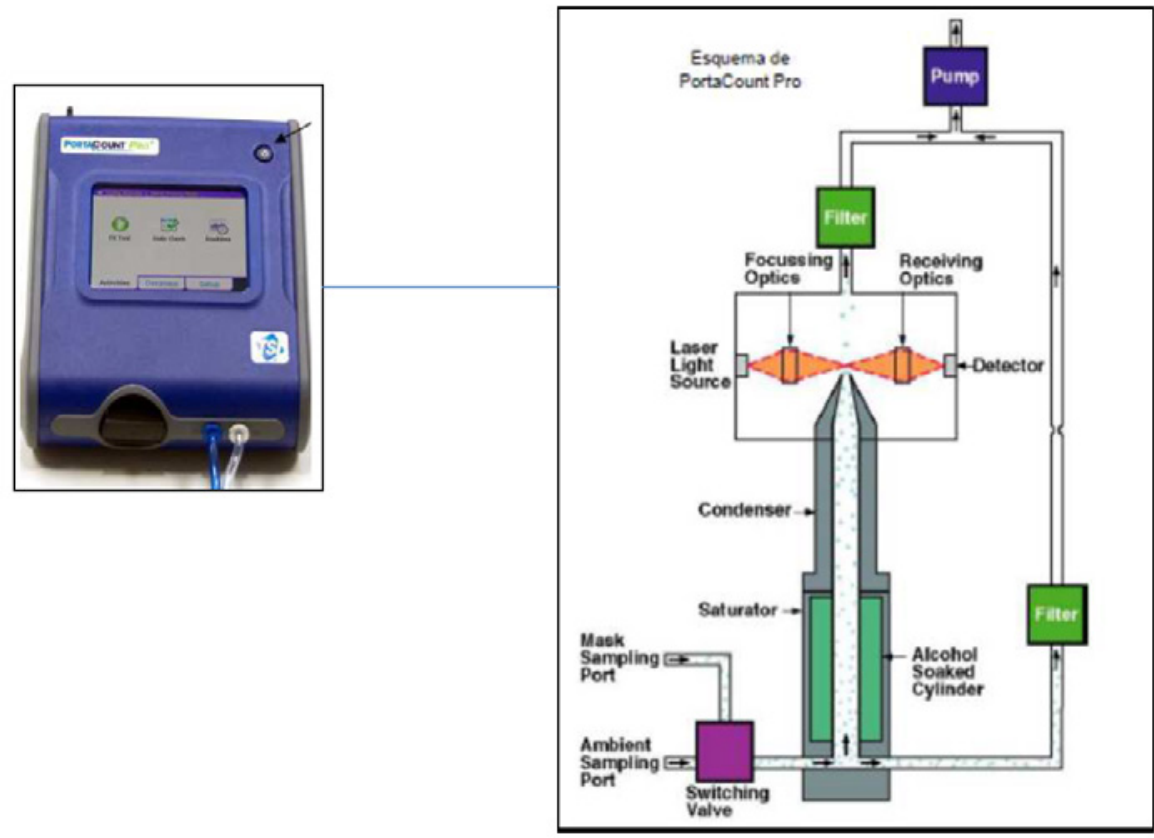

Figura 1: PortaCount ${ }^{\circledR}$ Pro+ Respirator Fit Tester 8038. 
El recuento de partículas está determinado por el recuento del número de impulsos generados durante un período de tiempo determinado. Puesto que conocemos el número de partículas, el período de tiempo y el caudal, podemos calcular la concentración de partículas. Las pruebas de ajuste cuantitativas se pueden llevar a cabo con cualquier aerosol, incluido el aire ambiente.

Para dicho análisis se ha realizado un test que está compuesto por las diferentes 7 pruebas (Figura 2) respiración normal, respiración profunda, movimiento de cabeza de lado a lado, de arriba a abajo, hablar o leer en voz alta, respiración normal para finalizar como la primera prueba, tocar o tratar de tocar los pies con las manos (o prueba alternativa).

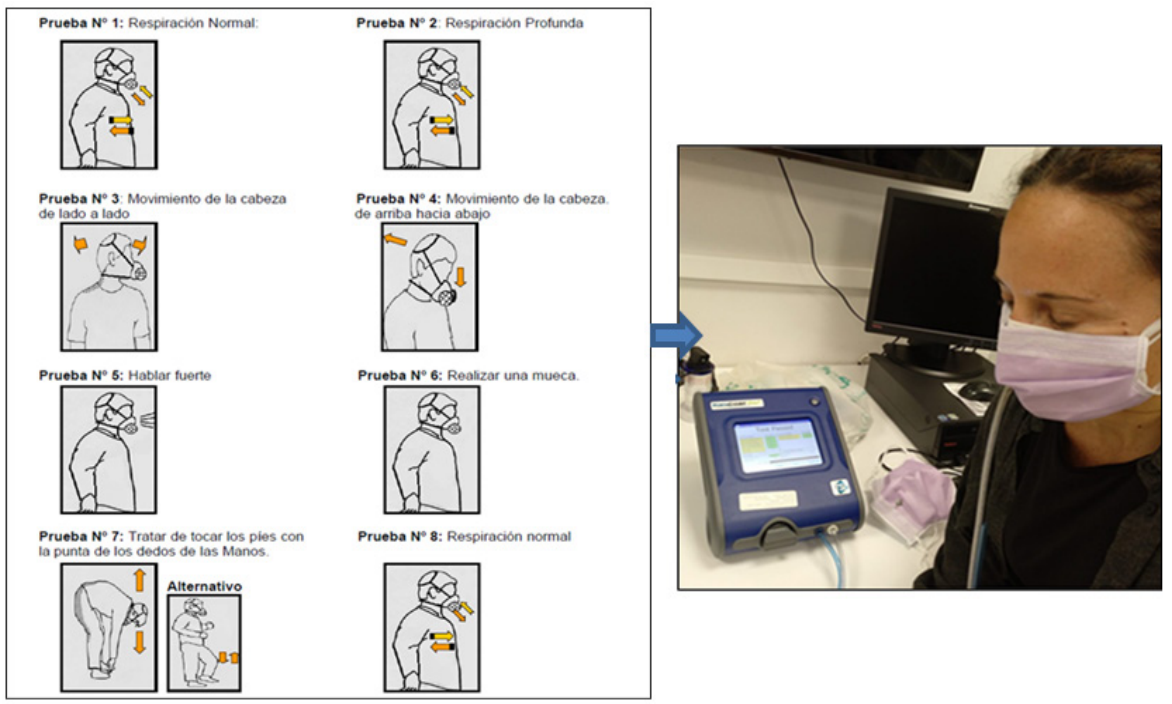

Figura 2: Diferentes pruebas del Test usados en el experimento.

Los modelos analizados de mascarillas autofiltrantes FFP2 para los test han sido (Figura 3).

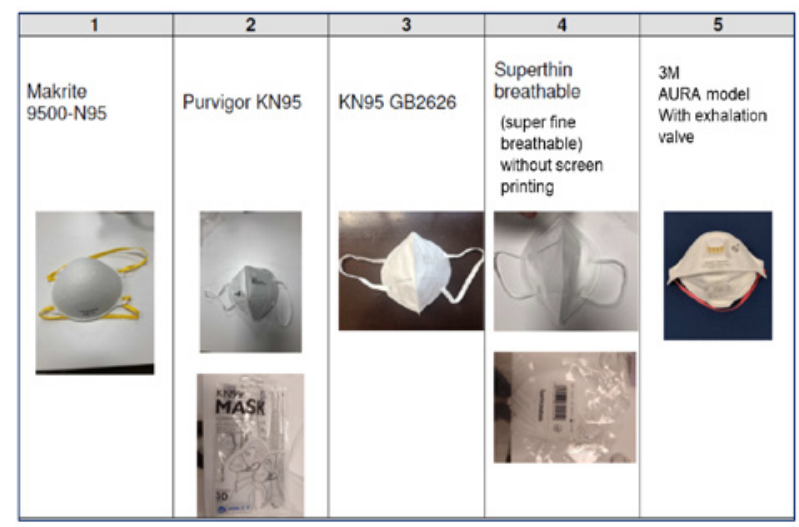

Figura 3: Modelos analizados de mascarillas autofiltrantes. 
Los modelos analizados de mascarillas quirúrgicas para el test han sido (Figura. 4).

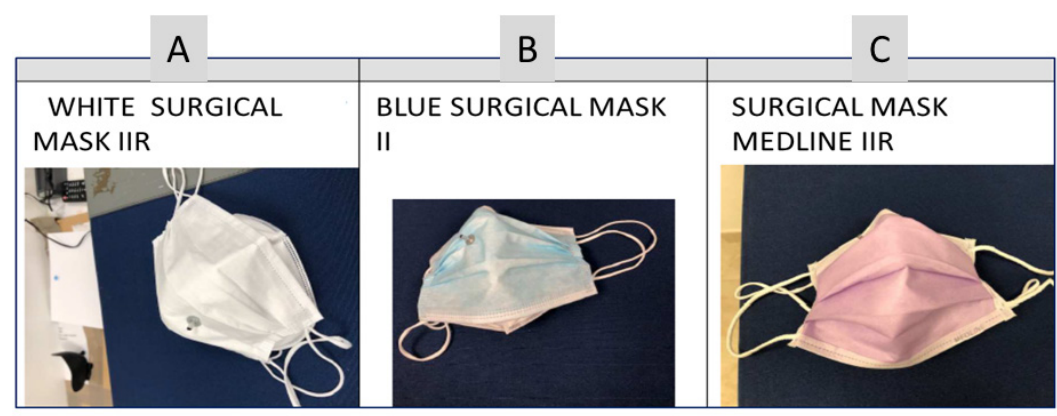

Figura 4: Modelos analizados de mascarillas quirúrgicas

\section{Resultados}

Los resultados que se han obtenido, buscando siempre el mejor ajuste posible y con la mejor protección a los riesgos ambientales al que está expuesto, se presentan en la Tabla 1.

Tabla 1: Resultados de los factores de ajuste para las diferentes mascarillas autofiltrantes.

\begin{tabular}{|c|c|c|c|c|c|}
\hline \multirow[b]{2}{*}{$\begin{array}{l}\text { Ejercicios Test con control de presión } \\
\text { negativa }\end{array}$} & \multicolumn{5}{|c|}{ Mascarillas FFP2 } \\
\hline & 1 & 2 & 3 & 4 & 5 \\
\hline 1-Respiración normal & 2,5 & 0,6 & 4,9 & 195 & $200+$ \\
\hline 2-Respiración profunda & 2,8 & 1,6 & 2,5 & 22 & $200+$ \\
\hline $\begin{array}{l}\text { 3-Movimientos de cabeza de lado a } \\
\text { lado }\end{array}$ & 4,6 & 9,6 & 5,8 & 5,2 & 182 \\
\hline $\begin{array}{l}\text { 4-Movimientos de cabeza de arriba a } \\
\text { abajo }\end{array}$ & 5,2 & 21 & 2,3 & 14 & 106 \\
\hline 5-Hablar o leer en voz alta & 26 & 14 & 6,1 & 25 & 74 \\
\hline 6-Flexión anterior de tronco & 6,6 & 18 & 5,1 & 50 & 66 \\
\hline 7-Respiración normal & 2,8 & 9,0 & 2,8 & 42 & 75 \\
\hline $\begin{array}{l}\text { Factor de ajuste } \\
\text { (valor de adecuación 100) }\end{array}$ & 4,1 & 2,6 & 3,6 & 17 & 105 \\
\hline $\begin{array}{l}\text { Valores de concentración en el } \\
\text { exterior/interior }\end{array}$ & $507 / 172$ & $389 / 43$ & $420 / 145$ & $444 / 10$ & $448 / 5,9$ \\
\hline
\end{tabular}


Los valores de las casillas son los factores de ajuste de las mascarillas obtenidos a través del test de ajuste. Son promedios de mediciones de concentraciones obtenidos a partir de las series por las mediciones realizadas. Las unidades de los valores de concentración son partículas $/ \mathrm{cm}^{3}$, pero al realizar el cociente para los factores de ajuste es adimensional. El factor de ajuste global se calcula a partir de los valores de ajuste individuales de cada ciclo de pruebas según la fórmula:

Factor de Ajuste Global $(F F g)=N$ / [(1/FF1)+(1/FF2)+(1/FF3) $+\ldots+(1 / F F n-1)+(1 / F F n)]$

$$
N=\text { Número de ejercicios realizados. }
$$

$F F n=$ Factor de Ajuste obtenido en el número de ejercicio que corresponda

Los valores son los que se obtienen en el registro de las pruebas sin posibilidad de escoger el mejor o peor valor. Se ha realizado una serie que consta de 8 pruebas aunque en el estudio experimental no nos ha permitido realizar la prueba de la mueca y por lo tanto han resultado 7 pruebas. Para estos modelos de mascarilla solamente pasa el test de ajuste y filtrado la mascarilla FFP2 de la marca 3M ( $\left.n^{0} 5\right)$.

Los resultados obtenidos tras la combinación de diferentes mascarillas quirúrgicas con las autofiltrantes FFP2 se muestran en la tabla 2.

Tabla 2: Factores de ajuste para la combinación de diferentes mascarillas quirúrgicas con las autofiltrantes FFP2.

\begin{tabular}{|c|c|c|c|c|c|}
\hline \multirow[b]{2}{*}{ Pruebas según protocolo } & \multicolumn{5}{|c|}{ Mascarillas FFP2 + Mascarilla quirúrgica } \\
\hline & $2+\mathrm{A}$ & $2+B$ & $2+C$ & $4+C$ & $1+\mathrm{C}$ \\
\hline 1-Respiración normal & 80 & 118 & $200+$ & $200+$ & 16 \\
\hline 2-Respiración profunda & 66 & 102 & $200+$ & $200+$ & 20 \\
\hline $\begin{array}{l}\text { 3-Movimientos de cabeza de } \\
\text { lado a lado }\end{array}$ & 60 & 108 & $200+$ & $200+$ & 64 \\
\hline $\begin{array}{l}\text { 4-Movimientos de arriba a } \\
\text { abajo }\end{array}$ & 39 & 92 & 155 & $200+$ & 21 \\
\hline 5-Hablar & 32 & 62 & $200+$ & $200+$ & 53 \\
\hline 6-Flexión anterior de tronco & 14 & 7,6 & $200+$ & $200+$ & 44 \\
\hline 7-Respiración normal & 20 & 7,9 & $200+$ & $200+$ & 69 \\
\hline $\begin{array}{l}\text { Factor de ajuste } \\
\text { (valor de adecuación de 100) }\end{array}$ & 32 & 22 & 192 & $200+$ & 30 \\
\hline
\end{tabular}

Como no se pudieron hacer los test para todas las combinaciones, se eligieron aquellos que se utilizaban más y que en principio podrían dar mejores resultados. La mascarilla de 3M como el valor de ajuste era correcto, se consideró innecesario realizar el test con la misma. Para estos modelos de mascarilla en combinación con la quirúrgica sólo pasaría el test las mascarillas 2 y 4 si son combinadas con la mascarilla quirúrgica Medline " $\mathrm{C}$ ".

A la hora de utilizar una mascarilla es imprescindible tener en cuenta la fisonomía, el rostro, ya que debe ajustarse perfectamente para evitar que el aire que pueda 
estar contaminado pase entre la cara y la mascarilla. En este sentido, la mascarilla rígida 1, al no ajustarse correctamente a una fisonomía delgada (cara alargada), no ha pasado ningún test, ni utilizándose sola, ni en combinación con la quirúrgica. Este diseño de mascarillas sólo es recomendable para personas de cara redonda y en actuaciones de bajo riesgo de exposición.

Se identifica que hay mascarillas autofiltrantes que podrían no proteger completamente al trabajador de las partículas en suspensión de menor tamaño con carga viral viable por falta de ajuste. Dado que el coronavirus SARS-Cov-2 se transmite por aerosoles, estas mascarillas se pueden utilizar durante actuaciones de bajo riesgo.

Para las actuaciones de alto riesgo, donde el contaminante biológico puede estar en el ambiente como los aerosoles, si se utilizan mascarillas autofiltrantes, priorizar las mascarillas que hayan pasado los ensayos europeos de la norma UNE-EN 149:2001+A1:2009, con o sin válvula de exhalación. En el caso de los aerosoles, es preferible que sean FFP3, de no haber en stock o no disponer el profesional de ellas, utilizar una FFP2 con mascarilla quirúrgica aporta una protección equivalente (+200). En el caso de utilizar mascarillas con válvula de exhalación, siempre debe ponerse encima una mascarilla quirúrgica para evitar que el aire exhalado por dicha válvula pueda contaminar a los pacientes.

En este estudio se observó que determinados modelos de mascarillas autofiltrantes, que por sí mismas mostraron un grado de ajuste bajo o muy bajo, al combinarse con un modelo de mascarilla quirúrgica conseguían tal mejora de grado ajuste que éste alcanzaba valores óptimos (FFP2/N95 con goma auricular en combinación con mascarilla quirúrgica de Medline). Esta mejora no ocurría con todos los modelos de mascarilla quirúrgica.

\section{Discusión}

En general los factores de ajuste de las mascarillas autofiltrantes 1, 2 y 4 mejoraron significativamente con cualquier modelo de mascarilla quirúrgica. Se observa que los valores de factor de ajuste de las mascarillas autofiltrantes eran muy bajos, por ejemplo los modelos 2 y 4 . Pero al combinarse concretamente con la mascarilla quirúrgica del modelo C, daban valores cercanos o superiores a 200 para casi todas las situaciones de respiración ensayadas. Dicho aumento del factor de ajuste no fue tan espectacular al combinar con la mascarilla quirúrgica del modelo A y B.

Se debe prestar especial atención a los meticulosos pasos de uso del equipo de protección personal por parte de los trabajadores de la salud para prevenir la infección por SARS-COV2. Según el ECDC(13), es importante el uso apropiado de las mascarillas autofiltrantes, ya que el uso incorrecto de las mismas puede no conferir una protección eficaz. La mascarilla autofiltrante debe cubrir completamente la cara desde el puente de la nariz hasta el mentón. 
Roberge et al. ${ }^{(25)}$ demostraron que el uso de la mascarilla quirúrgica como barrera exterior sobre N95 FFR o N95 FFR/EV por parte de los trabajadores de la salud durante 1 hora a cualquiera de las dos tasas de trabajo asociadas con el entorno de la atención sanitaria, no provocaba un impacto fisiológico adicional significativo con respecto al de la FFR por sí sola. Dado que gran parte de la carga fisiológica asociada con el uso del FFR es atribuible a la necesidad de superar la resistencia del medio filtrante al flujo de aire; se esperaría que las capas adicionales de la mascarilla quirúrgica sobre un N95 FFR tuvieran un efecto aditivo sobre la resistencia respiratori. Sin embargo, la resistencia a la respiración de la mascarilla quirúrgica de tipo Il es bastante baja ( $<3 \mathrm{~mm}$ de presión de H2O) y, cuando se usa sobre diferentes modelos de N95 FFR con volúmenes de respiración bajos a moderados, se ha demostrado anteriormente que sólo da lugar a un aumento adicional del 4,6 al $10 \%$ en la resistencia a la inhalación y del 5,7 al 12,3\% en la resistencia a la exhalación ${ }^{(7)}$.

Un estudio reciente ${ }^{(26)}$ en pacientes con coronavirus estacionales ha demostrado que las mascarillas quirúrgicas redujeron significativamente la detección de ácido ribonucleico (ARN) viral en aerosoles y mostró una tendencia a reducir el ARN viral en gotas. Un reciente meta-análisis de ensayos controlados aleatorios ${ }^{(27)}$ también mostró que las mascarillas quirúrgicas son tan eficaces como las mascarillas N95 para reducir la transmisión de enfermedades similares a la gripe. Un meta-análisis de estudios observacionales proporcionó pruebas de efecto protector tanto de las mascarillas quirúrgicas como de las FFP2 o N95 contra el síndrome respiratorio agudo severo (SARS) y para el Covid-19 en los trabajadores de salud durante la atención médica que no genera aerosoles ${ }^{(28)}$.

Una mascarilla FFP2 o FFP3 (con una equivalencia aproximada a las mascarillas N95) es un dispositivo respiratorio diseñado para lograr un ajuste facial muy cercano y una filtración muy eficiente de las partículas transportadas por el aire. Están diseñadas para formar un sello alrededor de la nariz y la boca. Deben ser probados y no se puede lograr un ajuste adecuado en hombres con barba.

Cada vez más, el uso de máscarillas faciales se está convirtiendo en la nueva norma en nuestras vidas. En un intento por frenar la propagación del virus, más de 50 países han hecho que las mascarillas sean obligatorias en los espacios públicos durante la pandemia de COVID-19, como China, Singapur, España y Francia ${ }^{(29)}$. Las personas que usan mascarillas quirúrgicas corren el riesgo de exposición a los aerosoles a través de las partes laterales y superiores de la mascarilla facial. Por el contrario las mascarillass N95, FFP2, etc., tienen mucho mejor ajuste y son más estancas, lo que garantiza la protección.

Una vez colocada la mascarilla, esta no debería tocarse hasta el momento de su retirada, sin embargo es poco probable que esto suceda durante toda la jornada de trabajo del personal sanitario. Para solucionar el ajuste y el problema que se produce al tocar la mascarilla se puede usar una media de nylon u otra mascarilla de éste material por encima de la quirúrgica o la FFP2 o N95. Esta acción cumple tres funciones: mejora el problema del ajuste entre la cara y la mascarilla, evita que toquemos la mascarilla para recolocarla y mejora la eficacia de filtración ${ }^{(30)}$. 
Existe un comportamiento diverso entre distintos modelos de mascarillas autofiltrantes en cuanto al grado de ajuste al trabajador que las lleva puestas. Hay algún modelo de mascarilla autofiltrante que por sí misma proporciona un grado de ajuste óptimo (FFP2 modelo 3M). Hay otros modelos de mascarillas autofiltrantes que por sí mismas ofrecen un ajuste deficiente permitiendo el paso de un porcentaje importante de partículas del exterior al interior (algunas probadas equivalentes a N95 con goma auricular).

Si las mascarillas autofiltrantes no disponen de válvula de exhalación, utilizar una mascarilla quirúrgica preserva la durabilidad de la mascarilla que se posee debajo y mejora el ajuste, evitando así la entrada de cualquier tipo partículas que puedan contener microorganismos, incluído el SARS COV-2 del exterior al interior. Para las mascarillas con válvula de exhalación, las razones de utilizar una mascarilla quirúrgica serían tres: para preservar la durabilidad de la mascarilla, para mejorar el ajuste y para evitar que quien la lleve pueda contaminar a su entorno.

Actuales y futuras lineas de investigación en cuanto a las características de las mascarillas y su uso se encaminan al estudio sobre el diseño, durabilidad, comodidad, transpirabilidad así como el poder de filtración de las mismas ${ }^{(31)}$.

\section{Conclusiones}

La lucha contra cualquier enfermedad infecciosa requiere esfuerzos y soluciones en la prevención, detección, diagnóstico y tratamiento. Por lo tanto, el uso de máscarillas sirve como una estrategia clave para la prevención de enfermedades transmitidas por el aire que no puede sustituirse fácilmente. Según los resultados de este estudio, se podría afirmar que las mascarillas quirúrgicas mejoran significativamente el grado de ajuste de todas las mascarillas autofiltrantes cuando se usan conjuntamente. En base a los resutados de este estudio, las mascarillas quirúrgicas, cuando se utilizan conjuntamente con las mascarillas autofiltrantes, proporcionan una mayor eficacia de filtración. Los resultados de este estudio señalan que las mascarillas quirúrgicas utilizadas conjuntamente con las mascarillas autofiltrantes podrían proporcionar una mayor protección al usuario frente a la exposición a aerosoles.

\section{Responsabilidades Éticas}

El mencionado estudio ha sido aprobado por el Comité Etica Investigación Clinica del Hospital General de Catalunya-Grupo Hospitalario Quirón Salud. Sant Cugat del Vallés. Barcelona. España.

\section{Conflicto de interés}

Los autores declaran no tener ningún conflicto de intereses de ninguna índole.

\section{Financiación}

Este trabajo no ha recibido financiación de ningún tipo.

\section{Confidencialidad de los datos}

Los autores declaran que han seguido los protocolos de su centro de trabajo sobre la publicación de datos de pacientes. 


\section{Bibliografía}

1. Li Q, Guan X, Wu P, Wang X, Zhou L, et al. Early Transmission Dynamics in Wuhan, China, of Novel Coronavirus-Infected Pneumonia. N Engl J Med. 2020;382(13):1199-1207.

2. Bai Y, Yao L, Wei T, Tian F, Jin DY, et al. Presumed Asymptomatic Carrier Transmission of COVID-19. JAMA. 2020;323(14):1406-1407.

3. Kai-Wang To K, Tak-Yin Tsang O, Leung WS, Tam AR, Wu TC, et al. Temporal profiles of viral load in posterior oropharyngeal saliva samples and serum antibody responses during infection by SARS-CoV-2: an observational cohort. Lancet Infect Dis. 2020;20(5):565-574.

4. Wölfel R, Corman VM, Guggemos W, Seilmaier M, Zange S, et al. Virological assessment of hospitalized patients with COVID-2019. Nature. 2020;581(7809):465469.

5. Zhang J, Litvinova M, Wang W, Wang Y, et al. Evolving epidemiology and transmission dynamics of coronavirus disease 2019 outside Hubei province, China: a descriptive and modelling study. Lancet Infect Dis. 2020;20(7):793-802.

6. Zou L, Ruan F, Huang M, Liang L, Huang $H$, et al. SARS-CoV-2 viral load in upper respiratory specimens of infected patients. New Engl J Med. 2020;382(12):11771179.

7. Chatterjee P, Nagi N, Agarwal A, Das B, Banerjee S, et al. The 2019 novel coronavirus disease (COVID-19) pandemic: A review of the current evidence. Indian J Med Res. 2020;151(2-3):147-159.

8. World Health Organization (WHO). Rational use of personal protective equipment for COVID-19 and considerations during severe shortages: interim guidance. Consultado 4 Julio 2020. Disponible en: https://apps.who.int/iris/handle/10665/338033

9. Tartari E, Hopman J, Allegranzi B, Gao B, Widmer A, et al. Perceived challenges of COVID-19 infection prevention and control preparedness: A multinational survey. J Glob Antimicrob Resist. 2020;22:779-781.

10. Lee K, Shukla V, Clark M, Mierzwinski-Urban M, Pessoa-Silva CL, Conly J. Physical interventions to interrupt or reduce the spread of respiratory viruses - resource use implications: a systematic review. CADTH Technol Overv. 2012;2(3):e23

11. Chang $D, X u H$, Rebaza A, Dela Cruz CS. Protecting health-care workers from subclinical coronavirus infection. Lancet Respir Med. 2020;8(3):e13.

12. World Health Organization (WHO). Mask use in the context of COVID-19.1 December 2020 WHO/2019-nCoV/IPC_Masks/2020.5. Consultado 4 Julio 2020.

13. Disponible en: https://apps.who.int/iris/bitstream/handle/10665/331493/ WHO-2019-nCoV-IPC_Masks-2020.2-eng.pdf 
14. European Centre for Disease Prevention and Control (ECDC). Personal protective equipment (PPE) needs in healthcare settings for the care of patients with suspected or confirmed 2019-nCoV. Eur Cent Dis Prev Control Pers Prot Equip needs Healthc settings care patients with suspected or confirmed 2019-nCoV ECDC Stock 2020. Consultado 4 Julio 2020. Disponible en: https://www.ecdc.europa.eu/ en/publications-data/personal-protective-equipment-ppe-needs-healthcare-settings-care-patients\#no-link

15. Wizner $K$, Nasarwanji M, Fisher $E$, et al. Exploring respiratory protection practices for prominent hazards in healthcare settings. J Occup Environ Hyg. 2018;15(8):588-597.

16. Dharmadhikari AS, Mphahlele M, Stoltz A, Venter K, Mathebula R, et al. Surgical Face Masks Worn by Patients with Multidrug-Resistant Tuberculosis Impact on Infectivity of Air on a Hospital Ward. Am J Respir Crit Care Med. 2012;185(10):1104-9.

17. Maclntyre $C$, Chughtai $A$, Seale $H$. Uncertainty, risk analysis and change for Ebola personal protective equipment guidelines. Int J Nurs Stud. 2015;52(5):899903.

18. Macintyre R, Chughtai AA. For personal use only Facemasks for the prevention of infection in healthcare and community settings. BMJ. 2015;350:h694.

19. Cheng VC, Tai JW, Wong LM, Chan JF, Li IW, et al. Prevention of nosocomial transmission of swine-origin pandemic influenza virus $\mathrm{A} / \mathrm{H} 1 \mathrm{~N} 1$ by infection control bundle. J Hosp Infect. 2010;74(3):271-277

20. European Centre for Disease Prevention and Control (ECDC). Infection prevention and control and preparedness for COVID-19 in healthcare settings - Fifth update. Consultado 6 Octubre 2020.Disponible en : https://www.ecdc.europa.eu/ sites/default/files/documents/Infection-prevention-and-control-in-healthcare-settings-COVID-19_4th_update.pdf

21. Norma UNE-EN 149:2001+A1:2010. Dispositivos de protección respiratoria. Medias máscaras filtrantes de protección contra partículas. Requisitos, ensayos, marcado. Consultado 31 enero 2021. Disponible en: https://www.une.org/encuentra-tu-norma/busca-tu-norma/norma?c=N0044643

22. Lepelletier D, Grandbastien B, Romano-Bertrand S, Aho S, Chidiac C, et al. What face mask for what use in the context of COVID-19 pandemic? The French guidelines. J Hosp Infect. 2020;105(3):414-8.

23. Bollinger, Nancy US. NIOSH Respirator Selection Logic. October 2004. DHHS (NIOSH) Publication No. 2005-100. Consultado 31 enero 2021. Disponible en: https://www.cdc.gov/niosh/docs/2005-100/default.html

24. Ippolito M, Vitale F, Accurso G, lozzo P, Gregoretti C, et al. Medical masks and Respirators for the Protection of Healthcare Workers from SARS-CoV-2 and other viruses. Pulmonology. 2020;26(4):204-212. 
25. Roberge RJ, Coca A, Williams WJ, Palmiero AJ, Powell JB. Surgical mask placement over N95 filtering facepiece respirators: Physiological effects on healthcare workers. Respirology. 2010;15(3):516-21.

26. Chan J, Yuan S, Kok K, To K, Chu H, et al. A familial cluster of pneumonia associated with the 2019 novel coronavirus indicating person-to-person transmission: a study of a family cluster. Lancet [Internet]. 2020;395(10223):514-23.

27. Matthews R, Young A. Medical masks vs N95 respirators for preventing COVID-19 in healthcare workers: A systematic review and meta-analysis of randomized trials. Influenza Other Respir Viruses. 2020; 59(1):165-166.

28. Bartoszko JJ, Farooqi MAM, Alhazzani W, Loeb M. Medical masks vs N95 respirators for preventing COVID-19 in healthcare workers: A systematic review and meta-analysis of randomized trials. Influenza Other Respir Viruses. 2020;14(4): 365-373.

29. Aljazeera News. ¿Which countries have made wearing face masks compulsory? (17.08.2020). Consultado 31 enero 2021. Disponible en: https://www.aljazeera.com/news/2020/8/17/which-countries-have-made-wearing-face-masks-compulsory

30. Mueller AV, Eden MJ, Oakes JM, Bellini C, Fernandez LA. Quantitative Method for Comparative Assessment of Particle Filtration Efficiency of Fabric Masks as Alternatives to Standard Surgical Masks for PPE. Matter. 2020;3(3):950-962.

31. Chua MH, Cheng W, Goh SS, Kong J, Li B, et al. Face Masks in the New COVID-19 Normal: Materials, Testing, and Perspectives. Research (Wash D C). 2020;2020: 7286735. 


\section{Anexo 1. Certificado de Calibrado}

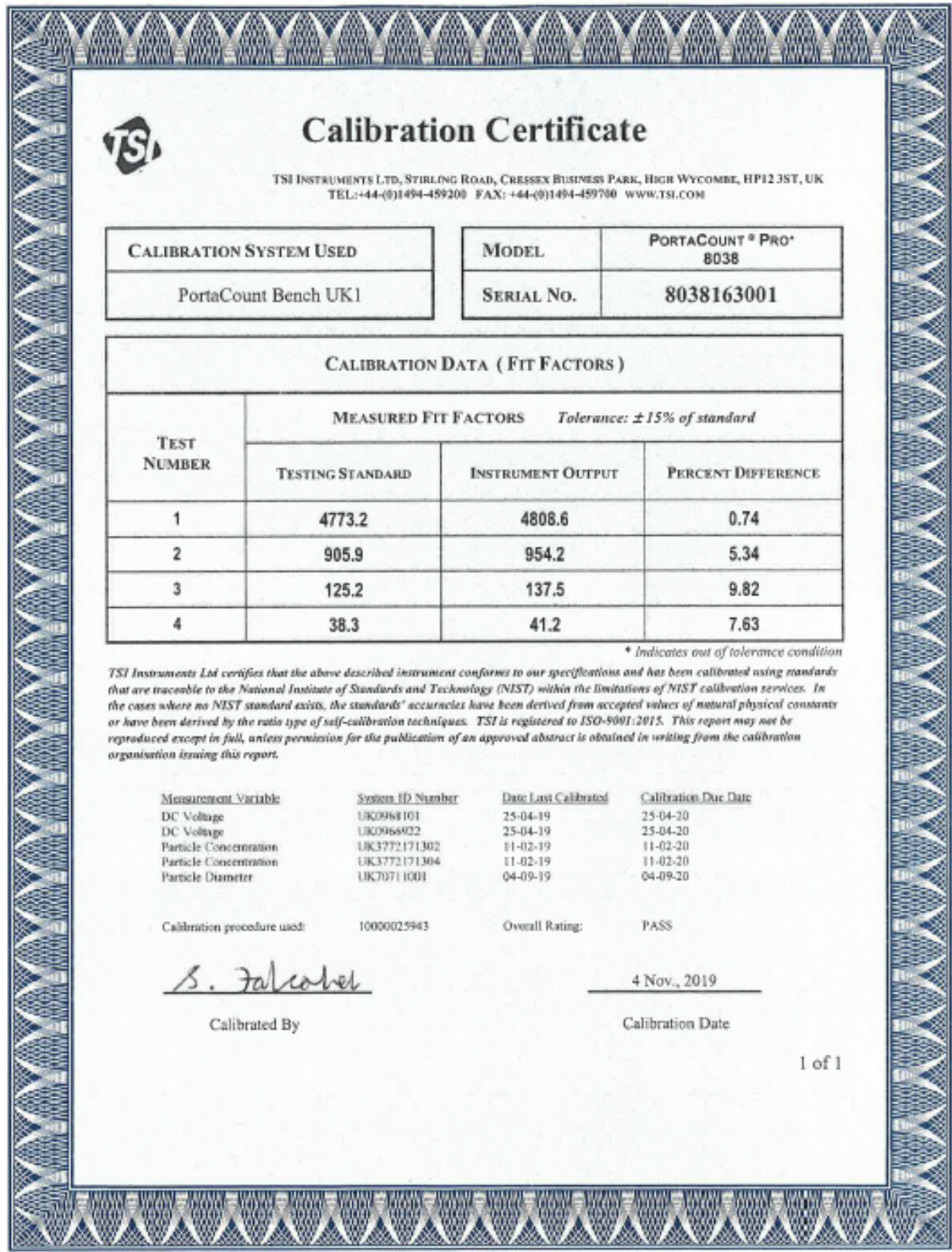

